\title{
Students teach students
}

\author{
A German-Japanese school education project based on a real time \\ audio-visual distance learning environment
}

\author{
Klaus-D Graf and Kiyoshi Yokochi \\ Freie Universitaet Berlin, Takustr. 9, 14195 Berlin, Germany. \\ graf@inf.fu-berlin.de \\ 4 of 8, 2-chome, Matsugaoka, Nakano-ku, Tokyol65 Japan. \\ Tokai University, Japan (professor emeritus) and \\ Beijing Normal University, China (visiting professor). \\ yokochi@blue.ocn.ne.jp
}

\begin{abstract}
Distance learning experiments in mathematics and science education as well as in art education in Japan have initiated considerable increase in creativity of pupils, improvement of scholarship, and growth in understanding cultural characteristics of different regions. Based on these experiments, an environment for German-Japanese school education projects using real time interactive audio-visual distance learning between remote classrooms has been established. This has been done with elementary school children speaking in their native languages, translated by interpreters on the topics of symmetries in rectangles and stripes. It has been done with lower secondary students speaking in English on topics related to discovering, proving, and applying the Pythagorean Theorem. Another project is underway now with four pairs of Berlin and Tokyo schools, co-operating in environmental education, Japanese language education, literature (haiku), and music education. In these experiments, the pupils take over the teaching, a method that will be more and more important in the future of learning. Initiated and supervised by their teachers, the pupils select problems from some subject matter, they explore the Internet, and in groups of 2 or 3 they prepare teaching clips for their peers in the foreign country. This technique brings about both progress in personal learning as well as deep mutual understanding in difficulties and aptitudes of the learning partners.
\end{abstract}

Keywords: Collaborative learning, distance learning, videoconferencing, culture, creativity

The original version of this chapter was revised: The copyright line was incorrect. This has been corrected. The Erratum to this chapter is available at DOI: 10.1007/978-0-387-35403-3_29 


\section{BACKGROUND FOR THE PROJECT}

There were two rather different observations about new objectives of education at the beginning of work on our joint project: Yokochi was concerned with the growing demand for an improvement of the quality of mathematics education, naming intentions like originality, creativity, comprehensive uses, mathematical scholarship, etc. In this context appreciation and exploitation of different cultural characteristics as well as interdisciplinary components (mathematics and arts) played highly influential roles.

Reflecting activities for more general intentions like interdisciplinary and intercultural context within subject matter education, Graf had pointed out that decision makers in education agree that modern societies rely increasingly on larger numbers of individuals with high-level knowledge and skills at their disposal (Graf 1995, referring to Ruiz, 1993). This means, in addition to elementary cultural techniques such as reading, writing and calculating, school has to teach other skills such as analysing, abstracting and modelling. Conceptual and communication skills are also required, in the fields of production and service. For example, an increasing range of jobs depend on individuals who know about their responsibilities in a wide context. Often many of these people must be able to work in multidisciplinary teams.

When exchanging these observations, we agreed that in Japan and in Germany as well as in other countries there are challenges to societies by universal problems such as the world economy or environment. These problems are of extreme complexity and require immense international interaction. Any success is dependent on mutual understanding and acknowledgement of different traditions and attitudes related with problems like mass production, environmental protection, political, and social structures. Readiness and goodwill have to be developed in young people growing into these societies, so more attention has to be given to interdisciplinary and intercultural intentions in education. There are avenues in many subjects in school to pursue directions such as the ones discussed above and every school subject could contribute. In particular, information and communication technologies form a rich source for contributing to such mutual understanding, linked with subjects like mathematics or social science which carry the high-level knowledge and skills mentioned above. 


\section{BASIC STRUCTURE OF THE LEARNING AND TEACHING EXPERIMENTS}

Several distance learning experiments of Yokochi's research group, including pairs of classes from distant provinces in Japan had shown that mathematics education as well as science and art education could profit considerably from theses activities. This encouraged us to plan practical work growing out of the very general considerations extended above. In a series of distance learning experiments between school classes in Japan and Germany, it could be demonstrated that these activities form an excellent platform to promote objectives like the ones mentioned above in school education. Each experiment consisted of different activities of two partner school classes and their teachers, together with researchers in didactics at universities, extending over six to twelve weeks. The activities started with preparation of a project by researchers and teachers, contacts running via mutual visits in Germany and Japan or via e-mail, Internet homepages or airmail. After this, teachers in the two countries started working with their classes concurrently on the same topic, mostly taken from mathematics, social science (environmental problems) or arts.

Besides learning about the topic in different ways the students, in more or less co-operation with their teachers, started to prepare demonstrations for their peers about their findings and results. These were executed in several videoconferences, integrated in the total activity of $6-12$ weeks. Material was also forwarded by e-mail or fax or put in the respective homepages in the Internet. Some conferences consisted of teaching of the teachers about the mathematical background of some products generated (tiles with patterns e.g.), and sometimes groups of students did the teaching.

In addition to concentration on special subject matter, which was essential in our design of the learning units, there were passages in the activities, per e-mail or in the video conferences, where the students tried to find out more personal information about each other concerning pets, grading systems, views on the other countries or people, including the students themselves.

\section{FINDINGS FOR GENERAL AND SUBJECT MATTER EDUCATION}

It should be mentioned that our experiments did not only effect the learning and understanding of the students. At the same time, the researchers from teacher education as well as the teachers active in the preparation, 
execution, and evaluation of the distance learning experiments profited a lot. They had to reflect deeply on the educational theories they were relying on, so that they could be understood by their partners in the other country. Considerable feedback had to be given to make sure that the others' intentions had been understood. It became clear that understanding went far beyond the language problems. Still, using English to explain activities based on German or Japanese language was a problem in itself. The teachers had extra work to invest since they had to explain their project in their environment, for example to colleagues, school administrators and parents. They experienced that both sides were very ambitious to "perform" good presentations and they had to control these tendencies for the sake of allowing genuine learning processes.

Before we give detailed descriptions of students, contents, methods, media and other topics determining our learning units, we want to list some general findings.

- The students' interest in each project was great and held on for the whole period of the learning units and afterwards

- The students were planning with great zeal how to explain their results to the pupils in the other country

- They were very interested in learning and understanding what they were told by the other students and how others solved their problems

- They picked up their ideas and adapted them when doing their own work

- They developed a very general interest in the other environment and country, also in individual peers

- They discussed the project with other students and teachers from their schools, and with their parents and families

- In the sessions they were extremely attentive and ready to perform

- They were competing in a friendly way; when they put questions they reinforced good answers by cheering.

- Without problems in using the new technology they put direct questions to their peers in the other countries, forgetting about the distance

- They patiently waited for translations or - when English was the joint language of communication - for repetitions of statements and questions

We also had positive findings about special intentions and goals related

to the subject matter chosen. In most cases it was geometry. Learning progress in some specific matter was always the main purpose of the experiments. We took great care that neither just general intentions like discussed above dominated the activities nor mere use of technology.

Especially for mathematics education we had put up a list with aims for the reorganisation of this subject:

- Initiating mathematical originality and creativity of students 
- Learning comprehensive uses of mathematics with other subjects through solving real problems

- Improving the mathematical scholarship of students

- Appreciating and acknowledging the mathematical cultural characteristics of each district or country in problem solving

- Cultural exchanges related to science and technique between students of two classes

- Interaction with peers from different regions in mathematical problem solving.

Interviews with the students as well as their behaviour and performance have shown that many elements of these purposes were attained through the intercultural learning units of our experiments. This confirms with the results reported by Yokochi and his project group about internal Japanese distance learning activities. Students took up contents as well as methods from their partners and used them when solving problems or creating art objects.

\section{AGE LEVELS AND CONTENT OF EXPERIMENTS}

The following partnerships and learning units between German and Japanese classes have been realised so far:

a) Peter-Witte-Primary-School in Berlin and Primary School attached to Yamanashi University in Kofu. The students came from grades 5 and 6 , there were 20 in Berlin and 40 in Japan. Their subject matter was stripe patterns and rectangular patterns, seen under aspects of mathematics and arts. There were four video conferences of one hour each included, two dominated by students presentations and discussions, two by teaching of the teachers to remote classes. Two German teachers performed team-teaching for some periods.

b) Hildegard-Wegscheider-Secondary-School in Berlin and Irihirose High School in Niigata. The students came from grade 10 in Berlin and grades 8 and 9 in Japan. Subject matter was the Theorem of Pythagoras. Groups of German students explained proofs on different levels: experimental work, visualisation by graphics and logical proofing based on constructions at the blackboard. This took about one hour.

c) Hildegard-Wegscheider-Secondary-School in Berlin and Secondary School attached to Yamagata University in Yamagata. The students came from grade 10 in Berlin and grade 9 in Japan. The subject matter 
was the sundial and its mathematical background from the geometry of the globe. Like in b) groups of Japanese students taught the German students using models and logical explanations at the blackboard. They put forward excellent questions to the German students and they showed great excitement on good answers.

The partners in subprojects d) to f) were secondary schools in Berlin and Tokyo. They had a common videoconference on one day, open to the public in Tokyo. The contributions were co-ordinated and prepared in a period of about five weeks.

d) Hildegard-Wegscheider-Schule and Mukogaoka School. Seven Japanese students gave a presentation of the City of Tokyo. 15 German students answered with singing a popular Japanese song and some of them then presented some facts and pictures from Berlin in the Japanese Language.

e) Canisius-College and Koyamadai School. Seven Japanese students gave a presentation about 'cleaning of the classroom by students', five German students answered about the same topic, followed by a lively discussion.

f) Camille-Claudel-Schule and Kokusai High School. Subject matter was "saving energy in school". Both groups of five students each presented explorations about the topic; the Germans reported about an investigation in their school. A lively discussion followed, including questions about how the students experience each other and what they think about the other country, respectively.

g) An experiment at university level was performed between a research lab of Mitsubishi Electric and Freie Universitaet Berlin. German students from mathematics, computer science and physics were instructed by researcher Dr. Toshio Ito about using computer simulation when designing kites, including video simulation using a computer. The conference was interactive so that students could ask each other questions.

\section{TEACHING AND LEARNING METHODS}

Different methods were applied and experienced. In the primary school experiment both sides followed a rather conservative mode of teachers on 
both sides controlling the performance of the classes and asking most of the questions. The pupils were given opportunities to present their works by prepared statements. Students and teachers spoke in their mothers' languages; they were translated consecutively by interpreters in both classrooms.

The Pythagoras experiment was characterised by independent teaching of German groups of students to the other side. They had explored the topic on the Internet and evaluated the results together with their teacher. The effect of learning was considerable on both sides, since there was a high motivation to good teaching and understanding. This experiment was also characterised by both classes speaking English. This caused many misunderstandings and repetitions. Using figures and visualisations helped relieve this situation.

The sundial experiment was also conducted in English with the Japanese students doing most of the teaching in small groups. This was an outstanding event in a Japanese classroom.

In the Berlin - Tokyo experiments all activities came from the students after having communicated before via electronic means. As to language there was a special situation since all of the German students had taken Japanese language courses in their schools. So they had prepared their statements in Japanese language which was happily accepted by their peers in Japan. Japanese students gave their contributions in their own language and the Germans tried to understand. Interpreters in both classrooms translated consecutively, if necessary.

After we had noticed that the teaching could be done very well by small groups of students, co-operating carefully in structuring subject matter and applying different methods (informal, formal), we started planning to organise bilingual groups to prepare elements of teaching. So far, however, this has turned out to be too difficult and could not yet be realised. The main reason was the great amount of communication in English via e-mail, which needs constant assistance by the teachers.

This brings us to some general remark about the role of teachers in our special learning setting. We started our experiments with teachers organising most of the planning and the execution of work in the classes. The students' roles were mostly to demonstrate what they had learned. Elder students then took over some of the teaching. This was still organised or "filtered" by the teachers. With growing experience, the students' demand grew to erase the filters totally, including the activities of preparing to teach and so to replace their own learning by preparing to teach. This, of course, causes a gain in active learning, but at the same time a loss in efficient learning. 


\section{SCHOOL ENVIRONMENT AND MEDIA}

Experiments such as those described in this paper can only be performed in a very friendly environment. It certainly became clear from the descriptions above that many questions about the "how" and "why" of such experiments will arise. To accept the answers, decision makers in education, responsible for the schools, teachers, parents, and sponsors have to be very far-sighted and open-minded.

Students and teachers will have to invest very much time for such projects, and they have to be very patient. Intercultural understanding and co-operation is very rewarding, but it is hard work at the same time. Language is one problem; different thinking and planning styles are others.

Cost for equipment is considerable and so is the telecommunication. The minimal equipment is a computer, a beamer plus a standard teleconferencing unit (about 2,000 \$US). If a computer and beamer are not available, a total of \$US 10,000 will do. Communication needs 2 ISDN lines carrying 64 kilobytes per second each. Considering the development in telephone cost, a price of one dollar per minute can be expected, even between continents.

The equipment that we used in Germany and Japan was sponsored by Mitsubishi Electric. The company had expanded the structure of a standard teleconferencing system considerably to meet the demands from two school classes "conferring" (Koizumi and others, 1999). So the systems used a 42inch monitor in the German classroom (about 20 people) or a LC-projector on an 80 -inch screen in a Japanese classroom (about 40 people).

There was a panorama camera and a motorised camera zooming and following individual persons or groups in each room. Video recorders recorded incoming and outgoing pictures and sound for later evaluation. A camera projector (stand camera) was used to transport any pictures and graphics to the remote monitors. Besides controlling the system, the PC could be used to create and transport computer screen images. For good sound transport a complicated system of ceiling microphones was installed. Besides a basic readiness to use such equipment for distance learning as indicated above the system demands technical and didactical or methodological knowledge about the best use from teachers and students. This implies in-service teacher training and better pre-service teacher education. 


\section{CONCLUSIONS AND VISIONS}

Neither the cost of the equipment and communication nor the extra amount of work related to the new media for distance learning should discourage us from integrating these media into the teaching and learning processes. This situation is comparable to the days when computers first entered schools and appeared uneconomical and unusable for education.

Teleconferencing systems or what will be developed from them will be a standard medium in schools within several years. They will not only be used for communication with peers, which we consider a very fruitful application, but also for communication with any point on the globe and anybody on the globe who can contribute to the demands of students to get information in an interactive way. Teachers will have to become advisers and supervisors for this learning by exploring. They have to prevent students from wasting too much time by unqualified exploring.

Distance learning is originated from the problem of physical distance in time and/or space between a class and a teacher, or, more general, between learners and knowledge bases including intelligence to interact - real or artificial. The more this intelligence and its means to interact (through multimedia) grow, the less the physical distance will matter. Instead, cultural distance as we used it in our experiments will become important for the learning process between learners and an intelligent source of knowledge. This distance can cause the learners to get more provoked to learn from a distant source, be it a group of peers or a knowledge base, to learn more through competing, or just through imitation and variation.

Putting individuals into school classes for learning may have economical reasons. But - each student coming from a different environment (the "culture of a family", e.g.) - it also means to initiate some kind of distance learning. This idea can be forwarded to the family of man.

\section{REFERENCES}

Downes, T. and Zammit, K. (2000) New literacies for connected learning in global classrooms, in Taylor, H. and Hogenbirk, P. (Eds.) The Bookmark of the School of the Future. Boston/Dordrecht/London: Kluwer Academic Publishers.

Graf, K-D. (1995) Promoting interdisciplinary and intercultural intentions through the history of informatics, in D. Watson and D. Tinsley (eds.) Integrating Information Technology into Education. London: Chapman \& Hall, 139-150.

Graf, K-D. and Yokochi, K. (1998) Educational experiments of distance learning and reorganisation of mathematics education, in D. Tinsley and D. C. Johnson (eds.) Information and Communication Technologies in School Mathematics. London: Chapman \& Hall, 265-274. 
Koizumi, K., Dasai, T., and Moriya, S. (1997) Proposal of interactive distance learning with $\mathrm{CCV}$ educational system and verification experiments. In Proceedings of the Int. Conference for Computer Communications (ICCC'97), 107-114,

Koizumi, H., Dasai, T., Yokochi, K., Graf, K-D, and Moriya, S. (1999) An interactive distance learning system and verification experiments between Japan and Germany, in D. Watson and T. Downes (eds.) Communications and Networking in Education. Boston/ Dordrecht/ London: Kluwer Academic Publishers, 39-50

Ruiz i Tarrago, F. (1993) Integration of information technology into secondary education: Main issues and perspectives, in Van Weert (ed.) Guidelines for Good Practice, IFIP Working Group 3.1, Geneva.

Wibe, J. and others (1999) Projects in Networked Learning: European experiences, in Watson, D. and Downes, T. [eds] Communications and Networking in Education. Boston/ Dordrecht/ London: Kluwer Academic Publishers, 101-126

\section{BIOGRAPHIES}

Klaus-D Graf studied mathematics, physics and education in Erlangen, Berlin, Muenchen, Tuebingen and Mainz between 1957 and 1996. He was a research assistant at the University of Illinois and the California Institute of Technology in 1963'64 before taking his $\mathrm{PhD}$ in Mathematics at the University of Mainz and becoming Assistant Professor in Cybernetics. He became Professor in mathematics and mathematics education in 1971, and subsequently in cybernetics, computer science, and computer science education at Freie Universitaet Berlin. In 1987 he was visiting professor at the National Central University at Taiwan. He specialises in applications of information and communication technologies in mathematics education, history of computer science, and international and intercultural Cupertino. $\mathrm{He}$ is an active member of IFIP working groups and ICMI activities.

Kiyoshi Yokochi has held professional posts at Wako University, Yamanashi and Tokai Universities before becoming guest professor at Beijing Normal University in 1985. He has been president of the Mathematical Education Society of Japan since 1986, organiser of the Five Nations Conference on Mathematics Education since 1986 and organiser of the International Conference on Cultural History of Mathematics since 1991. His special fields include mathematics, mathematics education, informatics, cultural history of mathematics, and kindergarten education. 